\title{
FINITELY GENERATED COHERENT ANALYTIC SHEAVES
}

\section{BERNARD KRIPKE}

If $S$ is a coherent analytic sheaf on the complex analytic space $X$, then for each $x \in X$, the stalk $\delta(x)$ is a finitely generated $\theta(x)$-module, where $\theta$ is the structure sheaf of $X[1]$. Since $\theta(x)$ is a local ring, there is a minimum number, \#( $\delta, x)$, of germs that generate $\delta(x)$ as an $\mathcal{O}(x)$-module, and every set of generators for $\mathcal{S}(x)$ contains a subset of $\#(S, x)$ generators $[2$, p. 14].

If there are $n$ global sections $s_{1}, \cdots, s_{n} \in \mathcal{S}(X)$ whose germs generate the stalk of $S$ at every point, then evidently:

(A) for every $x \in X, \delta(x)$ is generated by global sections of $\delta$ and,

(B) $\{\#(S, x): x \in X\}$ is a bounded set of integers. In fact, $\{\#(S, x): x \in X\}$ is bounded by $n$. The principal result of this note is that the converse also is true in case $X$ has finite global dimension. If the global sections of $\delta$ generate its stalk at each point and if $\{\#(S, x): x \in X\}$ is bounded, then finitely many of the global sections of $\mathcal{S}$ generate its stalk at each point.

Let us say that a subset $G$ of $s(X)$ generates $s \mid K$ if for each $x \in K$, $\{s(x): s \in G\}$ generates the stalk $\delta(x)$. If $K=X$, say that $G$ generates $s$. An ordered $n$-tuple $\left(s_{1}, \cdots, s_{n}\right) \in \delta\left(X^{n}\right)$ generates $s \mid K$ if $\left\{s_{1}, \cdots, s_{n}\right\}$ generates $\delta \mid K$. Let $G(S, n, K)$ be the set of all $n$-tuples in $\delta(X)^{n}$ which generate $\delta \mid K$.

If $U$ is an open subset of $X$, then $\delta(U)$ has a natural metrizable topology, which makes $\delta(U)$ into a Fréchet space. If $V$ is open and contains $U$, the restriction map $r_{V U}: S(V) \rightarrow S(U)$ is continuous [1, Chapter VIII]. A residual set in $\delta(X)^{n}$ is the complement of a set of the first category.

1. Theorem. Let $X$ be a d-dimensional analytic space and let $\mathcal{S}$ be a coherent analytic sheaf on $X$ that is generated by $\mathrm{S}(X)$. If \#(S, $x) \leqq n$ for every $x \in X$, then $G(S, n(d+1), X)$ is a dense residual set in $S(X)^{n(d+1)}$; in particular, it is not empty.

The theorem follows from a series of lemmas.

2. Lemma. Let $X$ be a complex analytic space and let $S$ be a coherent analytic sheaf on $X$. If $U$ is an Oka-Weil domain in $X[1, p .211]$ and $K$ is a compact $\mathcal{O}(U)$-convex subset of $U$, then $G(\mathcal{S}, n, K)$ is open in $S(X)^{n}$, for each positive integer $n$.

Received by the editors February 26, 1968. 
Proof. Suppose $t_{1}, \cdots, t_{n} \in \mathcal{S}(X)$ generate $s \mid K$. Then $K$ has an open neighborhood $V \subseteq U$ that is also an Oka-Weil domain such that $t_{1}, \cdots, t_{n}$ generate $\delta \mid V\left[1\right.$, pp. 211 and 244]. Identify $\mathfrak{C}^{n \times n}$ with the space of $n \times n$ complex matrices, and let $E: \mathcal{O}^{n \times n}\left|V \rightarrow \mathcal{S}^{n}\right| V$ be the map defined by $E(f)=\left(\sum_{j} f_{i j} t_{j}, \cdots, \sum_{j} f_{n j} t_{j}\right)$. Then $E$ is a homomorphism of coherent sheaves. Ker $E$ is a coherent sheaf, and by Cartan's Theorem $\mathrm{B}, H^{1}(V, \operatorname{Ker} E)=0$. Since $t_{1}, \cdots, t_{n}$ generate $s \mid V$, the sequence $0 \rightarrow H^{0}(V, \operatorname{Ker} E) \rightarrow H^{0}\left(V, \mathcal{O}^{n \times n}\right) \rightarrow H^{0}(V, \delta) \rightarrow 0$ is exact. That is, $E: \mathcal{O}(V)^{n \times n} \rightarrow \mathcal{S}(V)$ is a surjection. $R=\left\{f \in \mathcal{O}(V)^{n \times n}: f(x)\right.$ is an invertible matrix for each $x \in K\}$ is open in $\theta(V)^{n \times n}$, since the topology of $\mathcal{O}(V)^{n \times n}$ is that of uniform convergence on compacta [1, p. 237], and the set of invertible matrices is open in $\mathfrak{C}^{n \times n}$. It follows from the Open Mapping Theorem for Fréchet spaces that $E(R)$ is open in $S(V)^{n}$. Since $r_{X V}$ is continuous, $r_{X V}^{-1}(E(R))$ is open in $S(X)^{n}$. But if $s \in S(X)^{n}$ and $r_{X V}(s) \in E(R)$, then $s$ generates $s \mid V$. Thus $G(\mathcal{S}, n, K)$ contains a neighborhood $r_{\bar{X}}^{-1}(E(R))$ of $t$.

3. Lemma. Let $X$ be a complex analytic space, $S$ a coherent analytic sheaf on $X$, and $s_{1}, \cdots, s_{n} \in \delta(X)$. Then $Y=\left\{y \in X: s_{1}(y), \cdots, s_{n}(y)\right.$ do not generate $S(y)\}$ is an analytic subvariety of $X$.

Proof. Let $J$ be the subsheaf of $S$ generated by $s_{1}, \cdots, s_{n}$. Then $Y$ is the support of the coherent analytic sheaf $\mathcal{S} / \mathfrak{J}[3$, p. 87].

4. Lemma. Let $X$ be a complex analytic space, let $x \in X$, and let $\mathcal{S}$ be a coherent analytic sheaf on $X$ such that $\delta(X)$ generates $\delta(x)$. Let $n \geqq \#(S, x)$. Then $G(\mathcal{S}, n,\{x\})$ is dense in $S(X)^{n}$.

Proof. Since $\delta(X)$ generates $\delta(x)$ and $n \geqq \#(S, x)$, we can choose a $t \in \mathcal{S}(X)^{n}$ that generates $\delta(x)$. Let $s$ be any element of $\mathcal{S}(X)^{n}$. Say $s_{i}(x)=\sum_{j} c_{i j} t_{j}(x)$, where the matrix-valued function $c$ is analytic in a neighborhood of $x$. Then $s(x)-\lambda t(x)=(c-\lambda I) t(x)$, so that $s-\lambda t$ will generate $\delta(x)$ provided that the matrix $c-\lambda I$ is nonsingular in a neighborhood of $x$. This will be true if $\lambda$ is distinct from each of the $n$ eigenvalues of the matrix $c(x)$. There are arbitrarily small numbers $\lambda$ with this property. Hence there are sections $s-\lambda t$ of $s(X)^{n}$ arbitrarily close to $s$ that generate $\delta(x)$.

5. Lemma. Let $X$ be a d-dimensional complex analytic space, let $\$$ be a coherent analytic sheaf on $X$, and let $K$ be a compact subset of $X$. Suppose that $s(X)$ generates $s \mid K$ and that $n \geqq \#(S, x)$ for each $x \in K$. Then $G(\delta, n(d+1), K)$ is dense in $\delta(X)^{n(d+1)}$.

Proof. Let $A$ be a nonempty open set in $\delta(X)^{n(d+1)}$. Then $A$ contains a nonempty open set of the form $A_{1} \times \cdots \times A_{d+1}$, where $A_{i}$ 
is open in $S(X)^{n}$ for $i=1, \cdots, d+1$. Suppose that $0 \leqq k \leqq d+1$. Let us show that for each $i$ such that $1 \leqq i \leqq k$, we can choose a section $s^{i} \in A_{i}$ with the following property. Let $Y_{k}=\left\{x \in X:\left(s^{1}(x), \cdots, s^{k}(x)\right)\right.$ does not generate $\delta(x)\}$. ( $Y_{k}$ is a variety by Lemma 3.) Then no irreducible branch of $Y_{k}$ of dimension greater than $d-k$ intersects $K$.

The proof is by induction on $k$. For $k=0, Y_{0}=X$ and there is nothing to prove. Suppose we have chosen $s^{1}, \cdots, s^{k}$ so that no irreducible branch of $Y_{k}$ of dimension greater than $d-k$ intersects $K$. Let $B_{1}, \cdots, B_{p}$ be the irreducible branches of $Y_{k}$ which do intersect $K$. Since $S$ is coherent, there is actually a neighborhood $U$ of $K$ such that $\#(S, x) \leqq n$ for each $x \in U$. Therefore, for each $j=1, \cdots, p$, we can choose a regular point $x_{j} \in B_{j}$ such that $\#\left(S, x_{j}\right) \leqq n$. Then $D_{j}$ $=\left\{s \in \delta(X)^{n}: s\right.$ generates $\left.\delta\left(x_{j}\right)\right\}$ is open (Lemma 2) and dense (Lemma 4) in $\mathcal{S}(X)^{n}$. Choose $s^{k+1}$ in $A_{k+1} \cap D_{1} \cap \cdots \cap D_{p}$. Then no $(n-k)$-dimensional branch of $Y_{k+1}$ can intersect $K$.

In particular, $\left(s^{1}, \cdots, s^{d+1}\right)$ is an element of $A$ such that $Y_{d+1} \cap K$ $=\varnothing$, or in other words, $\left(s^{1}, \cdots, s^{d+1}\right)$ is an element of $A$ that generates $s \mid K$.

Proof of Theorem 1. Express $X$ as the union of countably many compact subsets $K_{1}, K_{2}, K_{3}, \cdots$, each of which is contained in an Oka-Weil domain in which it is holomorphically convex. According to Lemmas 2 and $5, G\left(S, n(d+1), K_{j}\right)$ is open and dense in $\delta(X)^{n(d+1)}$ for each $j=1,2,3, \cdots$. Therefore,

$$
G(\mathcal{S}, n(d+1), X)=\bigcap_{j=1}^{\infty} G\left(\mathcal{S}, n(d+1), K_{j}\right)
$$

is a residual set in $S(X)^{n(d+1)}$. Since $S(X)^{n(d+1)}$ is a Fréchet space, the Baire Category Theorem shows that $G(\delta, n(d+1), X)$ is dense in $\mathcal{S}(X)^{n(d+1)}$.

6. Corollary. If $X$ is a d-dimensional analytic space and $B$ is a n-dimensional vector bundle over $X$ which is generated by its global sections, then $B$ is generated by $n(d+1)$ of its global sections.

If $X$ is a Stein space, every coherent sheaf on $X$ satisfies condition (A), according to Cartan's Theorem A [1]. At least when $X$ is an open subset of a Stein manifold, the converse is also true. Indeed, in this case $X$ satisfies the hypotheses of the following proposition, according to Rossi [4].

7. Proposition. Let $X$ be an analytic space with the following properties. 
(a) $X$ can be embedded as an open subset of a Stein space $Y$ in such a way that the restriction map $r: \mathcal{O}(Y) \rightarrow \mathcal{O}(X)$ is bijective.

(b) Whenever $S$ is the sheaf of ideals of a 0 -dimensional variety in $X$, $\delta(X)$ generates $\delta$.

Then $X$ is a Stein space.

Proof. It will be enough to show that $X=Y$. If $X \neq Y$, there must be a component $C$ of $Y$ which is not contained in $X$. However, $C \cap X$ cannot be empty since the restriction $\mathcal{O}(Y) \rightarrow \mathcal{O}(X)$ is injective. Therefore, there must be a $y$ on the boundary of $C \cap X$ with respect to $C$.

Let $\left\{x_{n}\right\}$ be a sequence in $C \cap X$ converging to $y$, and let $\delta$ be the sheaf of ideals of $\left\{x_{n}\right\}$ over $X$. Let $Z=\{x \in X: f(x)=0$ for each $f \in \mathcal{S}(X)\}$. It will be enough to show that $Z$ has dimension greater than 0 , for then we will have contradicted the hypothesis that $S$ is generated by its global sections. Since $r$ is a bijection, we can form $W$ $=\left\{w \in Y:\left(r^{-1} f\right)(w)=0\right.$ for each $\left.f \in S(X)\right\}$. Clearly, $W$ is a subvariety of $Y$ and $W \cap X=Z$. Since $y$ is an accumulation point of $Z, \operatorname{dim}_{y}(W)$ $\neq 0$. If the irreducible branches of $W$ passing through $y$ are $B_{1}$, $\cdots, B_{p}$, at least one of them must contain infinitely many points of $Z, B_{1}$ let us say. But then $\operatorname{dim} B_{1}>0$, and $\operatorname{dim} Z \geqq \operatorname{dim} B_{1}$.

8. Proposition. Let $M$ be a 2-dimensional complex manifold and let $f: \mathcal{O}^{n} \rightarrow \mathcal{O}^{m}$ be an $\mathcal{\theta}$-homomorphism. Then for every $x \in M, \#(\operatorname{Ker} f, x) \leqq n$.

Proof. Consider the exact sequence $(\operatorname{Ker} f)(x) \rightarrow \mathcal{O}(x)^{n} \stackrel{f}{\rightarrow} \mathcal{O}(x)^{m}$ $\rightarrow \mathcal{O}(x)^{m} /(\operatorname{Im} f)(x) \rightarrow 0$. According to the Hilbert Syzygy Theorem $[1$, p. 74], $(\operatorname{Ker} f)(x)$ is a free $\mathcal{O}(x)$-module. Since $(\operatorname{Ker} f)(x)$ is a free submodule of $\mathcal{\theta}(x)^{n}, \#(\operatorname{Ker} f, x) \leqq n$. (The field of quotients $Q$ of $\mathcal{O}(x)$, being the direct limit of copies of $\mathcal{O}(x)$, is a flat $\mathcal{O}(x)$-module. Thus the sequence $0 \rightarrow Q \otimes_{\mathcal{\Theta}(x)}(\operatorname{Ker} f)(x) \rightarrow Q \otimes_{\mathcal{\Theta}(x)} \mathcal{\Theta}(x)^{n}$ is exact. But $Q \otimes \mathcal{O}(x)^{n}$ is an $n$-dimensional vector space over $Q$, and $Q \otimes(\operatorname{Ker} f)(x)$ is a vector space over $Q$ of dimension \#(Ker $f, x)$.)

9. Corollary. Let $M$ be a 2-dimensional Stein manifold. If I and $J$ are finitely generated ideals in the ring of holomorphic complex-valued functions on $M$, then $I \cap J$ is also finitely generated.

Proof. The following proof was suggested to me by Lance Small. Let $a_{1}, \cdots, a_{n}$ be generators for $I$, and let $b_{1}, \cdots, b_{m}$ be generators for $J$. Let $g$ and $g$ be respectively the subsheaves of $\mathcal{O}$ generated by $I$ and $J$. According to Cartan's Theorem B, $I=\mathscr{g}(M), J=g(M)$, and $I \cap J=(\mathscr{g} \cap \mathcal{g})(M)$. Thus it will suffice to prove that $\mathscr{I} \cap \mathcal{g}$ is generated by finitely many of its global sections. In fact, it will be shown that 
$\#(\mathscr{g} \cap \mathcal{g}, x) \leqq n+m$ for every $x \in M$, and hence that $I \cap J$ is generated by $3(n+m)$ of its elements according to Theorem 1 .

Let $f: \mathcal{O}^{n+m} \rightarrow \mathcal{O}$ be the map defined by $f\left(c_{1}, \cdots, c_{n+m}\right)=c_{1} a_{1}$ $+\cdots+c_{n} a_{n}-c_{n+1} b_{1}-\cdots-c_{n+m} b_{m}$. Then $\#(\operatorname{Ker} f, x) \leqq n+m$ for every $x \in M$ by Proposition 8 . But the formula $\pi\left(c_{1}, \cdots, c_{n+m}\right)$ $=c_{1} a_{1}+\cdots+c_{n} a_{n}$ evidently defines a surjection $\pi: \operatorname{Ker} f \rightarrow g \cap \mathcal{g}$.

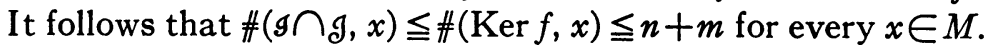

I do not know whether Corollary 9 would remain true if the condition of 2-dimensionality were dropped. I conjecture that it would not. In one dimension, the corollary is trivial since every finitely generated ideal is then principal.

10. Example. The bound $n(d+1)$ is the best possible if the density of $G(\mathcal{S}, n(d+1), X)$ in $\mathcal{O}(X)^{n(d+1)}$ is to be preserved.

Consider, in the complex plane $\mathcal{C}$, the subsheaf $\delta$ of $\mathcal{O}$ generated by the coordinate function $z$. Although one global section suffices to generate $\delta$, every section in a neighborhood of the section $z^{2}$ has two zeros in $\mathfrak{C}$, and therefore fails to generate $\mathcal{S}$.

\section{REFERENCES}

1. R. Gunning and H. Rossi, Analytic functions of several complex variables, Prentice-Hall, Englewood Cliffs, N. J., 1965.

2. M. Nagata, Local rings, Interscience, New York, 1962.

3. R. Narasimhan, Introduction to the theory of analytic spaces, Lecture notes in mathematics No. 25, Springer-Verlag, New York, 1966.

4. H. Rossi, On envelopes of holomorphy, Comm. Pure Appl. Math. 16 (1963), 9-19.

University of California, Berkeley 